\title{
Development and validation of the 3D U-Net algorithm for segmentation of pelvic lymph nodes on diffusion-weighted images
}

Xiang Liu' ${ }^{1}$, Zhaonan Sun ${ }^{1}$, Chao Han ${ }^{1}$, Yingpu Cui ${ }^{1}$, Jiahao Huang ${ }^{2}$, Xiangpeng Wang ${ }^{2}$, Xiaodong Zhang ${ }^{1}$ and Xiaoying Wang ${ }^{1 *}$

\begin{abstract}
Background: The 3D U-Net model has been proved to perform well in the automatic organ segmentation. The aim of this study is to evaluate the feasibility of the 3D U-Net algorithm for the automated detection and segmentation of lymph nodes (LNs) on pelvic diffusion-weighted imaging (DWI) images.

Methods: A total of 393 DWI images of patients suspected of having prostate cancer (PCa) between January 2019 and December 2020 were collected for model development. Seventy-seven DWI images from another group of PCa patients imaged between January 2021 and April 2021 were collected for temporal validation. Segmentation performance was assessed using the Dice score, positive predictive value (PPV), true positive rate (TPR), and volumetric similarity (VS), Hausdorff distance (HD), the Average distance (AVD), and the Mahalanobis distance (MHD) with manual annotation of pelvic LNs as the reference. The accuracy with which the suspicious metastatic LNs (short diameter $>0.8 \mathrm{~cm}$ ) were detected was evaluated using the area under the curve (AUC) at the patient level, and the precision, recall, and F1-score were determined at the lesion level. The consistency of $L N$ staging on an hold-out test dataset between the model and radiologist was assessed using Cohen's kappa coefficient.

Results: In the testing set used for model development, the Dice score, TPR, PPV, VS, HD, AVD and MHD values for the segmentation of suspicious LNs were $0.85,0.82,0.80,0.86,2.02(\mathrm{~mm}), 2.01(\mathrm{~mm})$, and $1.54(\mathrm{~mm})$ respectively. The precision, recall, and F1-score for the detection of suspicious LNs were 0.97, 0.98 and 0.97, respectively. In the temporal validation dataset, the AUC of the model for identifying PCa patients with suspicious LNs was 0.963 (95\% Cl: 0.892 0.993). High consistency of $L N$ staging $(K a p p a=0.922)$ was achieved between the model and expert radiologist.
\end{abstract}

Conclusion: The 3D U-Net algorithm can accurately detect and segment pelvic LNs based on DWI images.

Keywords: Prostate cancer, Lymph nodes, Segmentation, Detection, Diffusion-weighted imaging, Deep learning, U-Net

*Correspondence: wangxiaoying@bjmu.edu.cn

1 Department of Radiology, Peking University First Hospital, No.8 Xishiku

Street, Xicheng District, Beijing 100034, China

Full list of author information is available at the end of the article

\section{Background}

More than $15 \%$ of prostate cancer ( $\mathrm{PCa}$ ) patients were confirmed to have lymph node (LN) invasion during radical prostatectomy [1]. Patients with regional pelvic LN metastases face an increasing risk of mortality and should be treated aggressively [2]. Therefore, the detection of metastatic LNs is crucial for appropriate treatment selection and management. Precise LN staging allows 
urologists to determine which patients may benefit from a pelvic LN dissection (PLND) during radical prostatectomy and which patients may safely avoid it [3-5].

Multiparametric MRI (mpMRI) has been reported to play a central role in detecting and staging $\mathrm{PCa}[6$, 7]. Diffusion-weighted imaging (DWI) is characterized by a high contrast between the metastatic lesion and healthy tissue, thus yielding excellent efficiency in primary tumour evaluation and LN identification [8]. Radiologists usually regard LNs with a short diameter above $0.8 \mathrm{~cm}$ on DWI images as suspicious for metastatic LNs, and this should be highlighted in the radiology report [9, 10]. However, the detection of metastatic LNs on DWI images by radiologists is time-consuming and demands experience.

Recently, convolutional neural networks (CNNs) have emerged as a revolution in the field of image analysis. $\mathrm{CNN}$ is based on the concept that deep learning-based methods can provide an automated diagnosis and quantitative assessment with high reproducibility, thus allowing more objective reporting $[11,12]$.

Cuocolo et al. [13] compared different deep learning methods (U-Net, efficient neural network (ENet), and efficient residual factorized ConvNet (ERFNet)) for whole-gland and zonal prostate segmentation on T2WI images. Their results showed that ENet achieved the best Dice similarity score and U-Net for the second place. Comelli et al. [14] compared the performance of the U-Net and E-Net for lung segmentation on high-resolution computerized tomography images. The dice similarity coefficient showed no statistical differences between segmentation methods ( $95.9 \%$ vs. $95.61 \%, P=0.68$ ). Multiple studies have been published on the development of U-Net-based LN segmentation methods, most of which use CT $[15,16]$, PET/CT $[17,18]$, or MR lymphography images [19] rather than DWI images.

In this study, we introduced the 3D U-Net framework for automatically detecting and segmenting suspicious pelvic LNs in DWI images and then evaluated the framework on an hold-out test dataset. We hope that the automated detection and segmentation of LNs will lay a foundation for the comprehensive automated analysis of tumour burden in PCa patients.

\section{Materials and methods}

This retrospective study was performed with permission from the local institutional ethics committee. The need for written informed consent was waived.

\section{Study subjects}

A dataset of 425 patients suspected of having PCa between January 2019 and December 2020 was acquired from the local picture archiving and communication system (PACS) for algorithm development. The inclusion criteria were as follows: (1) patients with clinically suspected PCa (elevated PSA); (2) patients without any prior treatment of $\mathrm{PCa}$ (such as androgen deprivation, radical prostatectomy, or radiation therapy); and (3) patients whose high b-value $\left(1000\right.$ or $\left.800 \mathrm{~s} / \mathrm{mm}^{2}\right)$ DWI images were available. Fifteen patients were excluded due to unqualified MRI quality, 6 patients were excluded for obvious and massive metastatic involvement (e.g., obvious destruction of pelvic or bony structures), and 11 patients with a history of pelvic surgery were not included in the analysis. A total of 393 patients were finally recruited for 3D U-Net algorithm development, including 56 patients with PI-RADS scores of 1-2, 14 patients with PI-RADS scores of 3 , and 323 patients with PI-RADS scores of 4-5.

Another dataset of 77 patients with clinically suspected PCa seen between January 2021 and April 2021 was enrolled to externally evaluate the proposed algorithm. Among them, 37 patients had at least one suspicious metastatic LN (above $0.8 \mathrm{~cm}$ in the short-axis dimension and high signal intensity on DWI), and 40 patients did not have visible suspicious LNs. All mpMRI data were deidentified before inclusion, and the clinical information, such as age and prostate-specific antigen (PSA) level, of each enrolled patient was recorded.

\section{Pelvic mpMRI}

The mpMRI examinations were performed with three 3.0-Tesla scanners (Achieva, Philips Healthcare; Discovery, GE Healthcare; Interia, Philips Healthcare) using a phased-array coil. The standard mpMRI protocol at our institution included a combination of T2WI, T1WI, DWI, and dynamic contrast-enhanced imaging. Details of the imaging parameters of the DWI sequence are summarized in Table 1.

\section{Manual annotations}

Before annotation, all the Digital Imaging and Communication in Medicine (DICOM) format images were converted to NIFTI format. Images were manually annotated with ITK-SNAP (version 3.6; Penn Image Computing and Science Laboratory, Philadelphia, PA).

In the algorithm development dataset, all discernible LNs on DWI images were manually annotated by two junior radiologists (both with 4 years of reading experience) within the pelvic region (namely, Mask 1 and Mask 2). An expert radiologist (with more than 15 years of reading experience) subsequently modified the two sets of manual annotations (Mask 3 and Mask 4, respectively). The Dice score was used to evaluate the reliability of the manual annotations between different masks. 
Table 1 Imaging protocols of the pelvis DWI sequences

\begin{tabular}{llll}
\hline Protocols & $\begin{array}{l}\text { 3.0 T Achieva } \\
\text { (Philips Healthcare, the Netherlands) }\end{array}$ & $\begin{array}{l}\text { 3.0 T Discovery } \\
\text { (Ge healthcare, Milwaukee, WI) }\end{array}$ & $\begin{array}{l}\text { 3.0 T Interia } \\
\text { (Philips } \\
\text { Healthcare, the } \\
\text { Netherlands) }\end{array}$ \\
\hline B-values $\left(\mathrm{s} / \mathrm{mm}^{2}\right)$ & & 0,800 & 0,1000 \\
TR/TE $(\mathrm{ms})$ & 0,800 & $3000 / 60$ & $4959 / 78$ \\
Imaging matrix & $3400 / 54$ & $256 \times 256$ & $240 \times 240$ \\
Field of view $\left(\mathrm{mm}^{2}\right)$ & $224 \times 224$ & $360 \times 400$ & $360 \times 400$ \\
Slice thickness $(\mathrm{mm})$ & $375 \times 375$ & 8 & 7 \\
Number of slices & 6 & 25 & 28 \\
Intersection gap & 24 & No & No \\
\hline
\end{tabular}

TR Repetition time, TEEcho time

\section{Ground truth}

For model development, the manual annotations of the pelvic LNs were regarded as ground truth for the assessment of segmentation (Mask 4). LNs with short diameters $>0.8 \mathrm{~cm}$ were regarded as suspicious metastatic LNs, and their annotations were taken as ground truth for the assessment of LN detection (Fig. 1). For temporal validation, the manual annotations of the suspicious metastatic LNs, edited by one of the junior radiologists under the supervision of the expert radiologist, were regarded as ground truth for the segmentation and detection assessment. The LN staging performed by the expert radiologist was considered ground truth for the consistency evaluation of the $\mathrm{N}$-staging between the model and the radiologist.

\section{Model development}

The CNN developed for the segmentation of pelvic LNs on DWI images is the 3D U-Net [20], which replaced all $2 \mathrm{D}$ operations (convolution kernels, pooling layers, and

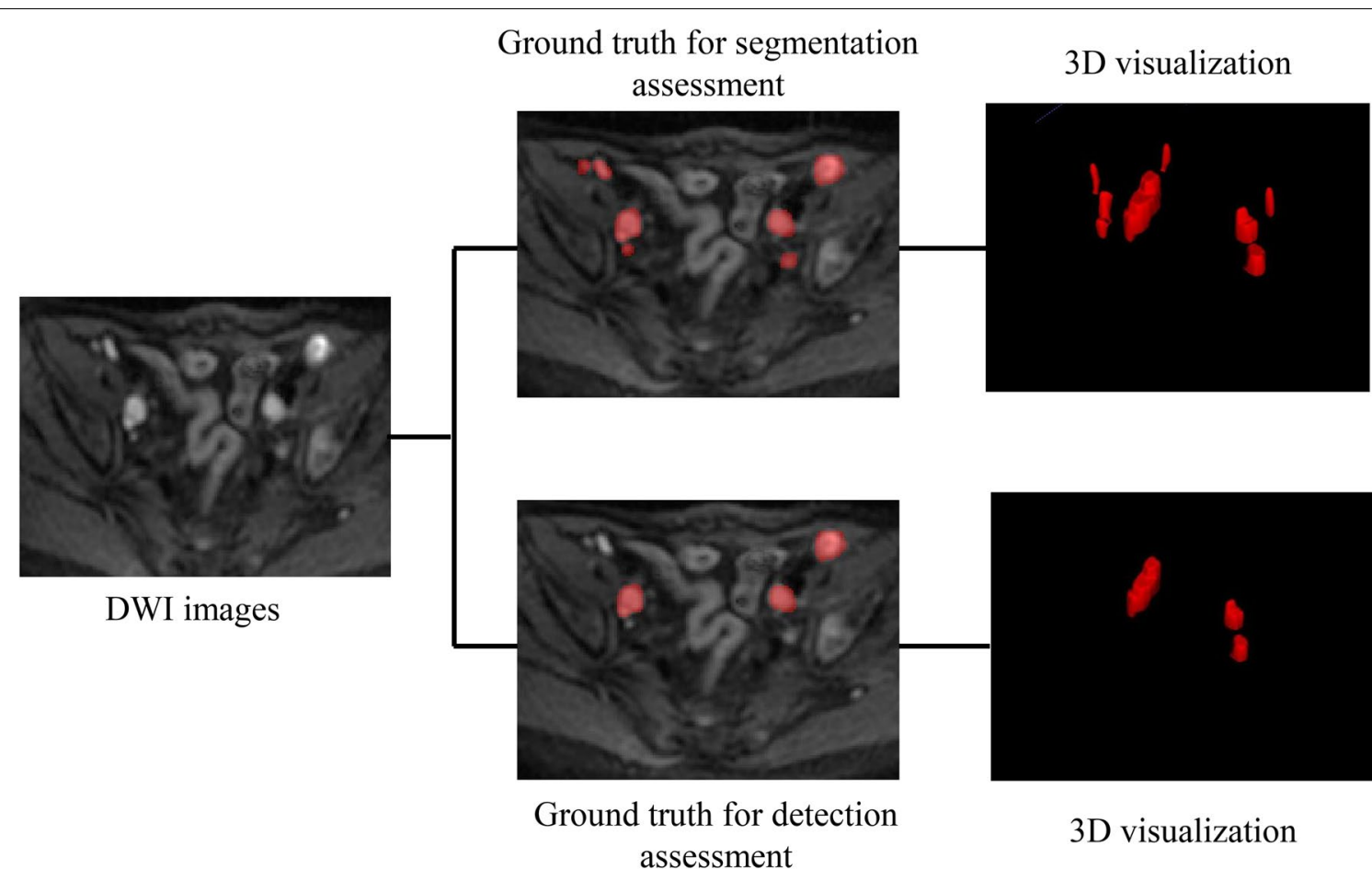

Fig. 1 Ground truth for model evaluation. The first row shows the ground truth for segmentation of the model, and the second row shows the ground truth for detection assessment of the model 
upconvolution kernels) of the U-Net architecture with 3D counterparts. By taking full advantage of the 3D spatial information, the algorithm can learn typical features with higher discrimination capability than those learned from 2D CNNs.

The 3D U-Net was trained with DWI images and their corresponding manual annotations. The 393 patients were randomly divided into the training $(\mathrm{n}=309)$, validation $(n=43)$ or testing $(n=41)$ set at a ratio of $8: 1: 1$. The hold-out test dataset $(n=77)$ was used to further evaluate the detection and segmentation accuracy of the 3D U-Net model (Fig. 2).
All input images were resized to $64 \times 256 \times 256(\mathrm{z}, \mathrm{y}, \mathrm{x})$ before training to maintain the optimal image features. To train the 3D U-Net segmentation models, we exploited the ADAM optimizer with an initial learning rate of $10^{-4}$ and a fixed batch size of 10 images. Skewing (angel: 0-5), shearing (angel: $0-5$ ) and translation (scale: $-0.1,0.1$ ) of the images were applied for data augmentation. U-Net was trained for 300 epochs until the validation loss function failed to rise. The network was written in Python (version 3.6). The PyTorch (version 0.4.1) deep learning platform was employed for training and validation. All experiments

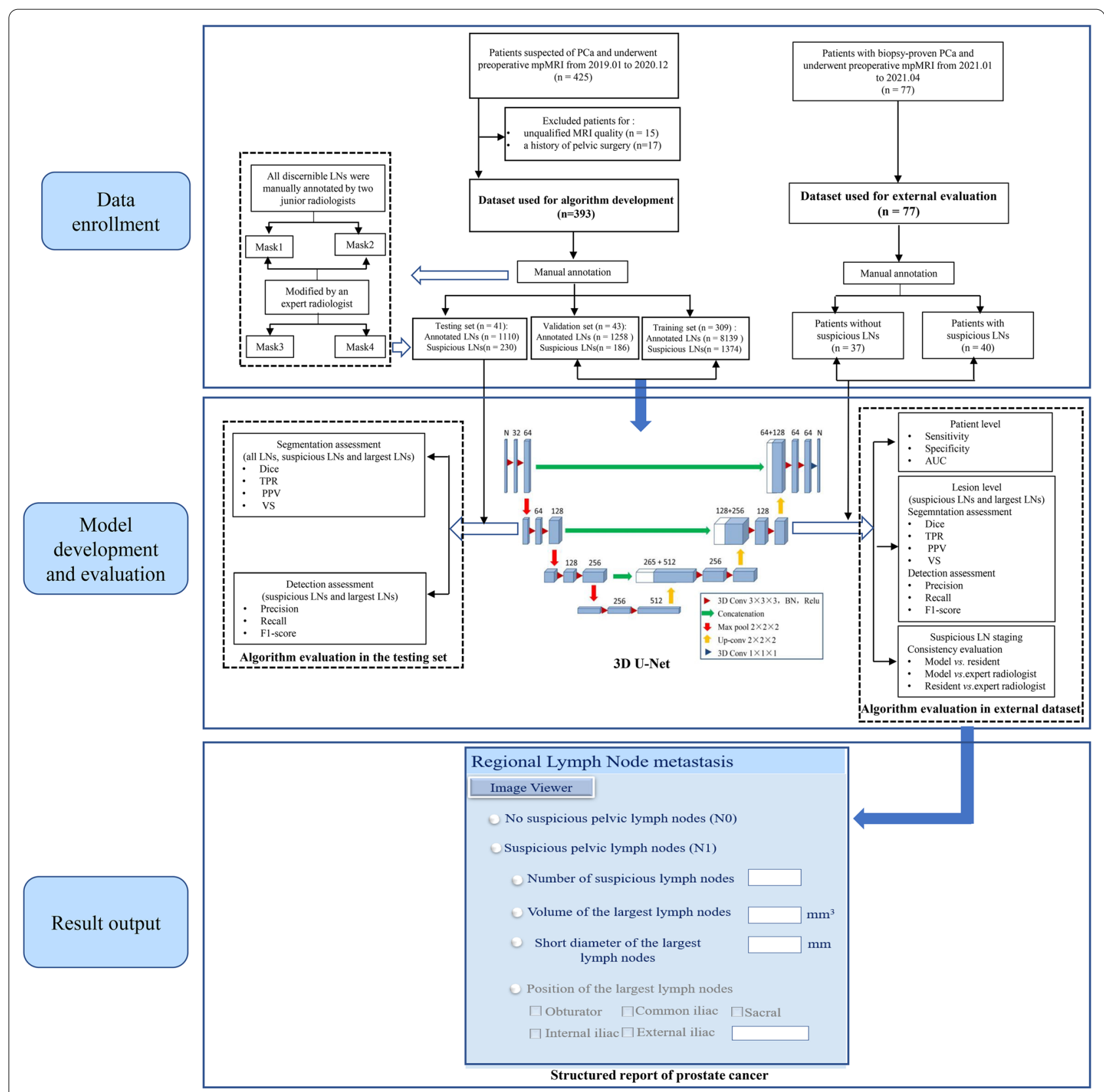

Fig. 2 The flowchart of the algorithm development and result output 
were performed using an NVIDIA Tesla P100 16G, running on Ubuntu.

\section{Quantitative measurement of LNs and output to the radiology report}

A cluster of contiguous voxels predicted by the algorithm was designated as an individual LN. After receiving the segmentation result of the $\mathrm{CNN}$, the volume and short diameter of each segmented LN were automatically calculated via the minimum-volume bounding box method. The number of suspicious LNs (short diameter $>0.8 \mathrm{~cm}$ ), as well as the volume and short diameter of the largest LN, were automatically sent to the structured report (Fig. 2). If at least one suspicious LN was detected by the model, then the N-staging was automatically inputted as N1 to the radiology report. In contrast, if no suspicious LN was detected, the N-staging was automatically inputted as N0 to the radiology report.

\section{Model performance evaluation}

Two datasets were collected in this study-the algorithm development dataset with manual annotations for all LNs and the hold-out test dataset with manual annotations only for suspicious LNs. Consequently, two sets of evaluation metrics were formulated to evaluate the algorithm.

\section{Evaluation using the model development dataset}

The ability of the 3D U-Net algorithm to segment and detect individual LNs was evaluated by comparison of the automated annotation with manual annotation. According to the overlap between manual annotation and automated segmentation, the voxels of the image can be defined as true positives (TPs), false positives (FPs), true negatives (TNs), and false negatives (FNs). The segmentation performance of the model was assessed using Dice, positive predictive value (PPV), true positive rate (TPR), volumetric similarity (VS), Hausdorff distance (HD), the Average distance (AVD), and the Mahalanobis distance (MHD) [21], which were defined by:

$$
\begin{aligned}
\text { Dice } & =\frac{2 T P}{2 T P+F P+F N} \\
P P V & =\frac{T P}{T P+F P} \\
T P R & =\frac{T P}{T P+F N} \\
V S & =1-\frac{|F N-F P|}{2 T P+F P+F N} \\
H D(A, B) & =\max (h(A, B), h(B, A)) \\
A V D(A, B) & =\max (d(A, B), d(B, A)) \\
M H D(X, Y) & =\sqrt{(\mu x-\mu y) T S-1(\mu x-\mu y)}
\end{aligned}
$$

where $h(A, B)$ is called the directed Hausdorff distance between two finite point sets $A$ and $B, d(A, B)$ is the directed Average Hausdorff distance between $A$ and $B, \mu x$ and $\mu y$ are the means of the point sets.

The average short diameter and volume of LNs in manual segmentation and automated segmentation were calculated to further quantitatively estimate the segmentation efficacy of the 3D U-Net algorithm.

Based on the segmentation results, we proposed a detection approach for suspicious LNs. A suspicious LN was considered to be correctly detected when overlap existed between automated segmentation and manual annotation. The precision, recall and F1-score (harmonic mean of precision and recall) were then calculated to evaluate the detection accuracy of the model. The precision and recall values reflected the percentage of correctly segmented LNs out of all the identified LNs $[\mathrm{TP} /(\mathrm{TP}+\mathrm{FP})]$ and the percentage of correctly segmented LNs out of all the annotated LNs $[\mathrm{TP} /(\mathrm{TP}+\mathrm{FN})]$, respectively. The segmentation performance (all LNs, suspicious LNs, and largest LNs) and detection performance (suspicious LNs and largest LNs) of the 3D U-Net were assessed in the testing set.

\section{Evaluation using the hold-out test dataset}

The hold-out test dataset was used to evaluate the ability of the model to discriminate between 37 patients with suspicious LNs and 40 patients without suspicious LNs. Sensitivity, specificity, and area under the receiver operating characteristic curve (AUC) were used to assess the performance of the algorithm at the patient level. Then, the segmentation performance (Dice, PPV, and TPR) and detection performance (precision, recall, and F1-score) of the model of suspicious LNs and largest LNs were assessed at the lesion level.

To further evaluate the clinical application value of the model of suspicious LN evaluation, the LN staging (N0 or N1) of the model was compared with the interpretation of the radiologists (one resident with less than 2 years of reading experience and one expert radiologist with more than 15 years of reading experience).

\section{Statistical analysis}

MedCalc (version 14.8; MedCalc Software, Ostend, Belgium) and SPSS (version 22.0, IBM Corp., Armonk, NY, USA) were used for the statistical analyses. Numerical data were averaged over all patients and reported as the mean \pm standard deviation (SD). One-way analysis of variance (ANOVA) was used to compare patient characteristics (age, PSA level, LN volume, and short 
diameter) and the segmentation performance of the algorithm (Dice, TPR, PPV, VS, HD, AVD and MHD) among different groups, and the least significant difference (LSD) was used for post hoc multiple comparisons. Paired $t$-tests, Pearson correlation, and Bland-Altman analyses were performed to compare the manual versus automated determination of the short diameter and volume of the LNs. Cohen's kappa coefficient was used to assess the consistency of LN staging between the model and the radiologists in the hold-out test dataset. $P<0.05$ was considered indicative of a statistically significant difference.

\section{Results}

Patient and LN characteristics

The patients' characteristics are presented in Table 2 . In the model development dataset, a total of 10,507 visible LNs were annotated: 8139 in the training set (27 per patient on average), 1258 in the validation set (29 per patient on average), and 1110 in the testing set (27 per patient on average). A total of 201 suspicious metastatic LNs were annotated in the hold-out test dataset of 37 PCa patients.

There was no significant difference in age or PSA level between the model development dataset and the holdout test dataset $(P=0.990$ and 0.088 , respectively). The PSA level between patients with or without suspicious LNs showed a significant difference $(P=0.031)$ in the hold-out test dataset. There was no significant difference in the number of annotated LNs, short diameter of the largest LNs, or volume of the largest LNs among the three sets in the model development dataset $(P=0.537$, 0.145 , and 0.628 , respectively).

\section{Reliability of the manual annotations}

The inter- and intrareader reliabilities of the manual annotations were assessed based on the Dice score. The Dice scores between different masks are as follows: Mask 1 vs. Mask 2: $0.75 \pm 0.03$; Mask 1 vs. Mask 3: $0.78 \pm 0.05$; Mask 2 vs. Mask 4: $0.80 \pm 0.09$; and Mask 3 vs. Mask 4: $0.88 \pm 0.06$. The high Dice scores between Mask 3 and Mask 4 confirmed the reliability of the manual annotations.

\section{Segmentation performance of the model}

The LN segmentation accuracy was evaluated in the testing set. As shown in Table 3, the model achieved optimal segmentation performance for the largest LNs with the highest Dice, TPR, PPV, VS, HD, AVD and MHD values of $0.88 \pm 0.15,0.89 \pm 0.21,0.83 \pm 0.16$, $0.88 \pm 0.20, \quad 2.02 \pm 0.09 \quad(\mathrm{~mm}), \quad 2.01 \pm 0.07 \quad(\mathrm{~mm})$, $1.54 \pm 0.12(\mathrm{~mm})$ respectively. The metrics of the segmentation accuracy of all LNs were significantly lower than those of the suspicious LNs and the largest LNs (all with $P<0.05$ ). There was no significant difference in the metrics between the suspicious and largest LNs (all with $P>0.05)$. There was no significant difference among different scanners concerning the segmentation accuracy of all LNs (all with $P>0.05$ ). Exemplary cases of segmentation of LNs with different Dice scores are shown in Fig. 3.

Table 2 The characteristics of patients and lymph nodes

\begin{tabular}{|c|c|c|c|c|c|c|c|c|}
\hline \multirow[t]{2}{*}{ Characteristics } & \multicolumn{4}{|c|}{ Model development dataset } & \multicolumn{3}{|c|}{ Hold-out test dataset } & \multirow[t]{2}{*}{$P$ value } \\
\hline & Training set & Validation set & Testing set & $P$ value & $\begin{array}{l}\text { Patients with } \\
\text { suspicious } \\
\text { LNs }\end{array}$ & $\begin{array}{l}\text { Patients } \\
\text { without } \\
\text { suspicious LNs }\end{array}$ & $P$ value & \\
\hline No. of patients & 309 & 43 & 41 & - & 37 & 40 & - & - \\
\hline Age, mean $\pm S D$ (years) & $70.3 \pm 9.6$ & $71.2 \pm 7.3$ & $70.8 \pm 8.8$ & 0.785 & $69.4 \pm 8.8$ & $71.4 \pm 8.4$ & 0.305 & 0.990 \\
\hline PSA, median (ng/ml) & $13.00(7.13,23.43)$ & $\begin{array}{l}14.21 \\
(8.20,26.73)\end{array}$ & $\begin{array}{l}12.20 \\
(7.42,21.30)\end{array}$ & 0.552 & $\begin{array}{l}15.49 \\
(9.25,26.65)\end{array}$ & $\begin{array}{l}10.69 \\
(7.10,18.82)\end{array}$ & 0.031 & 0.088 \\
\hline No. of annotated LNs & 8139 & 1258 & 1110 & - & - & - & - & - \\
\hline Average LNs per patient & $27(5,30)$ & $29(6,33)$ & $27(3,35)$ & 0.537 & - & - & - & - \\
\hline No. of suspicious LNs & 1374 & 186 & 230 & - & 201 & - & & \\
\hline Short diameter of largest LNs (cm) & $0.83 \pm 0.30$ & $0.93 \pm 0.33$ & $0.83 \pm 0.29$ & 0.145 & - & - & - & - \\
\hline Volume of largest LNs $\left(\mathrm{cm}^{3}\right)$ & $\begin{array}{l}9.03 \\
(4.60,17.32)\end{array}$ & $\begin{array}{l}10.93 \\
(5.30,17.40)\end{array}$ & $\begin{array}{l}7.09 \\
(5.37,14.08)\end{array}$ & 0.628 & - & - & - & - \\
\hline \multicolumn{9}{|l|}{ Scanners } \\
\hline 3.0 T Achieva & 96 & 16 & 12 & - & 12 & 15 & - & - \\
\hline 3.0 T Discovery & 113 & 13 & 15 & - & 14 & 13 & - & - \\
\hline 3.0 T Interia & 100 & 14 & 14 & - & 11 & 12 & - & - \\
\hline
\end{tabular}

PSA prostate-specific antigen, $L N$ lymph node 


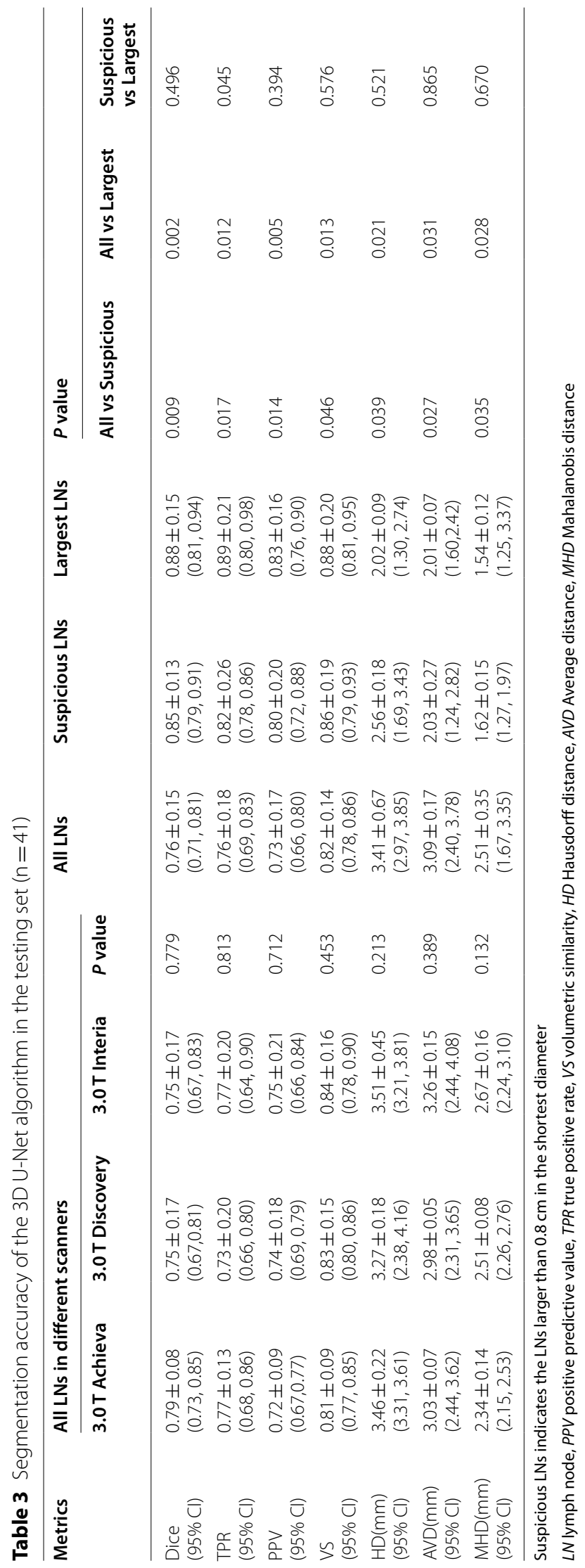




\section{Quantitative evaluation of segmentation performance} The average short diameter and volume measurements for all LNs, suspicious LNs, and largest LNs are summarized in Table 4. Quantitative comparisons between automated and manual segmentation are shown in Fig. 4. Both the short diameter and volume of the automatically segmented LNs showed a close correlation (all with $\mathrm{R}>0.80$ ) with manually annotated LNs. The Bland-Altman analysis of short diameter and volume showed good consistency between the automated segmentation and manual annotation of all LNs, suspicious LNs, and largest LNs, and most values were within the consistency interval.

\section{LN detection based on segmentation}

Detection of suspicious LNs and the largest LNs in the testing set are shown in Table 5. The developed method achieved good performance in the detection of suspicious LNs with a precision of $0.97(226 / 231)$, recall of $0.98(226 / 230)$, and F1-score of 0.97. Moreover, all 41 of the largest LNs from $41 \mathrm{PCa}$ patients were correctly detected by the algorithm.

Figure 5 illustrates examples of the detection results obtained with the developed method for LNs, which shows the TP (Fig. 5a), FP (Fig. 5b), and FN detections (Fig. 5c). Typically, FPs occur due to nonspecific high intensity; FNs usually occur with misattribution of small lesions and insufficient contrast compared with background.

\section{Segmentation and detection performance in the hold-out test dataset}

The hold-out test dataset was collected from a different reference period and there is no cross-contamination between model development dataset and hold-out test dataset. The segmentation and detection accuracy of the model in the hold-out test dataset were assessed at the patient and lesion levels. The sensitivity of the model in identifying PCa patients with or without a suspicious LN was $100 \%$ (37/37, 95\% CI: $90.5-100 \%)$, and the specificity reached 92.5\% (37/40, 95\% CI: 79.6-98.4\%), with an AUC of 0.963 (95\% CI: 0.892-0.993).

At the lesion level, the model's segmentation accuracy for suspicious LNs achieved an average Dice score, TPR, PPV, and VS of $0.83 \pm 0.15,0.80 \pm 0.04$, $0.81 \pm 0.07$, and $0.85 \pm 0.12$, respectively. Based on the segmentation result, the 3D U-Net achieved a detection precision of 0.96 , recall of 0.98 , and F1-score of 0.97 for suspicious LNs.

Cohen's kappa coefficients of the LN staging for the model and the resident and expert radiologist were as follows: model $v s$. expert radiologist: 0.922; model vs. resident: 0.766; and resident $v s$. expert radiologist: 0.844. The consistency between the model and the
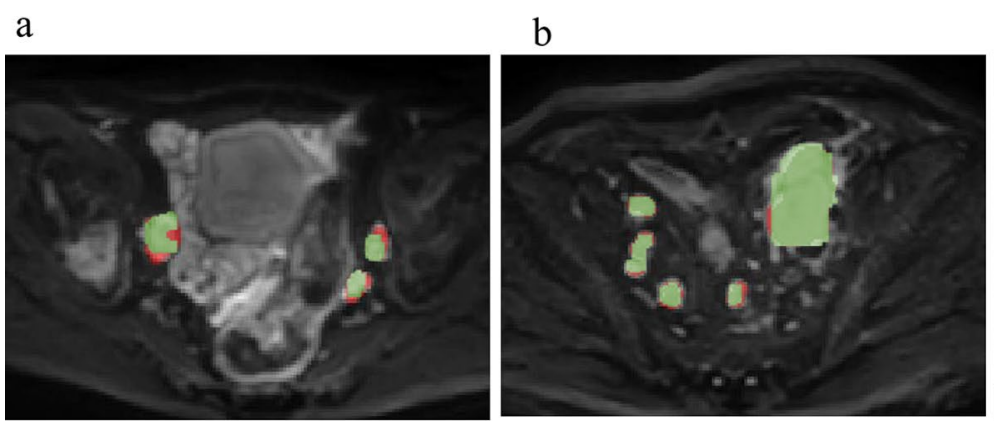

$\mathrm{C}$

Fig. 3 Examples of the segmentation results of the 3D U-Net for the lymph nodes. a The automated segmentation of LNs with a Dice score of 0.78; b The automated segmentation of $L N s$ with a Dice score of 0.85 ; c The automated segmentation of $L N s$ with a Dice score of 0.93 (red label: manual annotation; green label: automated segmentation)

Table 4 Quantitative measurements between automated segmentation and manual annotation in the testing set $(n=41)$

\begin{tabular}{|c|c|c|c|c|c|c|c|c|c|}
\hline \multirow{2}{*}{$\begin{array}{l}\text { Quantitative } \\
\text { metrics }\end{array}$} & \multicolumn{3}{|l|}{ All LNs } & \multicolumn{3}{|l|}{ Suspicious LNs } & \multicolumn{3}{|l|}{ Largest LNs } \\
\hline & $\begin{array}{l}\text { Automated } \\
\text { segmentation }\end{array}$ & $\begin{array}{l}\text { Manual } \\
\text { annotation }\end{array}$ & $P$ value & $\begin{array}{l}\text { Automated } \\
\text { segmentation }\end{array}$ & $\begin{array}{l}\text { Manual } \\
\text { annotation }\end{array}$ & $P$ value & $\begin{array}{l}\text { Automated } \\
\text { segmentation }\end{array}$ & $\begin{array}{l}\text { Manual } \\
\text { annotation }\end{array}$ & $P$ value \\
\hline Volume $\left(\mathrm{cm}^{3}\right)$ & $5.35 \pm 3.71$ & $5.45 \pm 2.79$ & 0.829 & $9.54 \pm 3.88$ & $10.09 \pm 2.66$ & 0.709 & $11.78 \pm 5.25$ & $11.67 \pm 6.04$ & 0.887 \\
\hline $\begin{array}{l}\text { Short diameter } \\
(\mathrm{cm})\end{array}$ & $0.46 \pm 0.30$ & $0.51 \pm 0.31$ & 0.482 & $0.95 \pm 0.29$ & $0.99 \pm 0.20$ & 0.492 & $1.12 \pm 0.50$ & $1.22 \pm 0.53$ & 0.810 \\
\hline
\end{tabular}

LN lymph node 


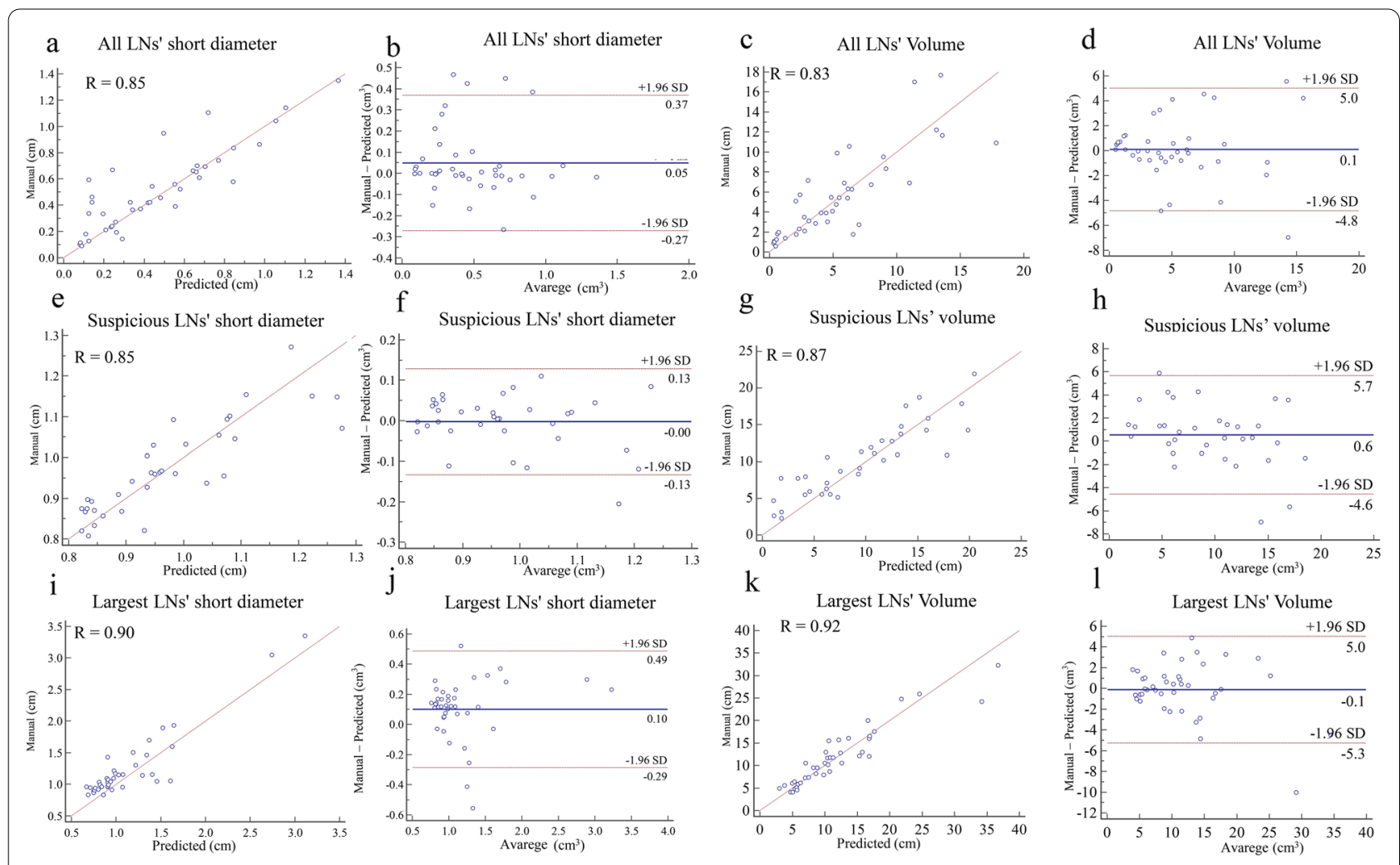

Fig. 4 Quantitative comparisons of the LNs'short diameter and volume. Correlation and Bland-Altman plots of LNs' short diameter and volume between automated segmentation and manual segmentation for all LNs (a-d), suspicious LNs (e-h), and largest LNs (i-I)

Table 5 Detection accuracy of lymph nodes in the testing set $(n=41)$

\begin{tabular}{lll}
\hline & Suspicious LNs & Largest LNs \\
\hline True positive & 226 & 41 \\
False Positive & 5 & 0 \\
False Negative & 4 & 0 \\
Precision & 0.97 & 1.00 \\
Recall & 0.98 & 1.00 \\
F1-score & 0.97 & 1.00 \\
\hline
\end{tabular}

LN lymph node

expert radiologist was higher than that between the resident and expert radiologist.

\section{Discussion}

In this study, we proposed to develop a 3D U-Net algorithm to achieve automated detection and segmentation of pelvic LNs on DWI images for clinically suspected PCa patients. The results in the testing set and hold-out test dataset confirmed the feasibility of automated LN detection and segmentation, which may aid in LN staging, quantitative measurements of tumour burden and image-guided treatment of $\mathrm{PCa}$ patients.

The U-Net algorithm has been widely used for organ and lesion segmentation on MRI images, such as prostate $[22,23]$ and prostate lesions [24]. However, this method is not easily applied to LN lesions. There is great heterogeneity in the shape and size of lymphadenopathies in the pelvis, making it difficult to discriminate true LN regions from other regions. Compared with that of the background, LN lesions usually account for a small part of the image volume, and this imbalance makes segmentation more difficult. Additionally, the number of FPs that contain nonspecific high-intensity mimics is considerably large, which usually results in lower specificity. Last but not least, the inter- and intraoperator variability of manual annotation remains a longstanding bottleneck for automated image segmentation [25], and there is currently no reliable substitute for manual labelling.

To overcome the imbalanced data problem, several loss functions have been implemented in other studies. For example, the Tversky loss function has been successfully used for the segmentation aneurysmal of the ascending 


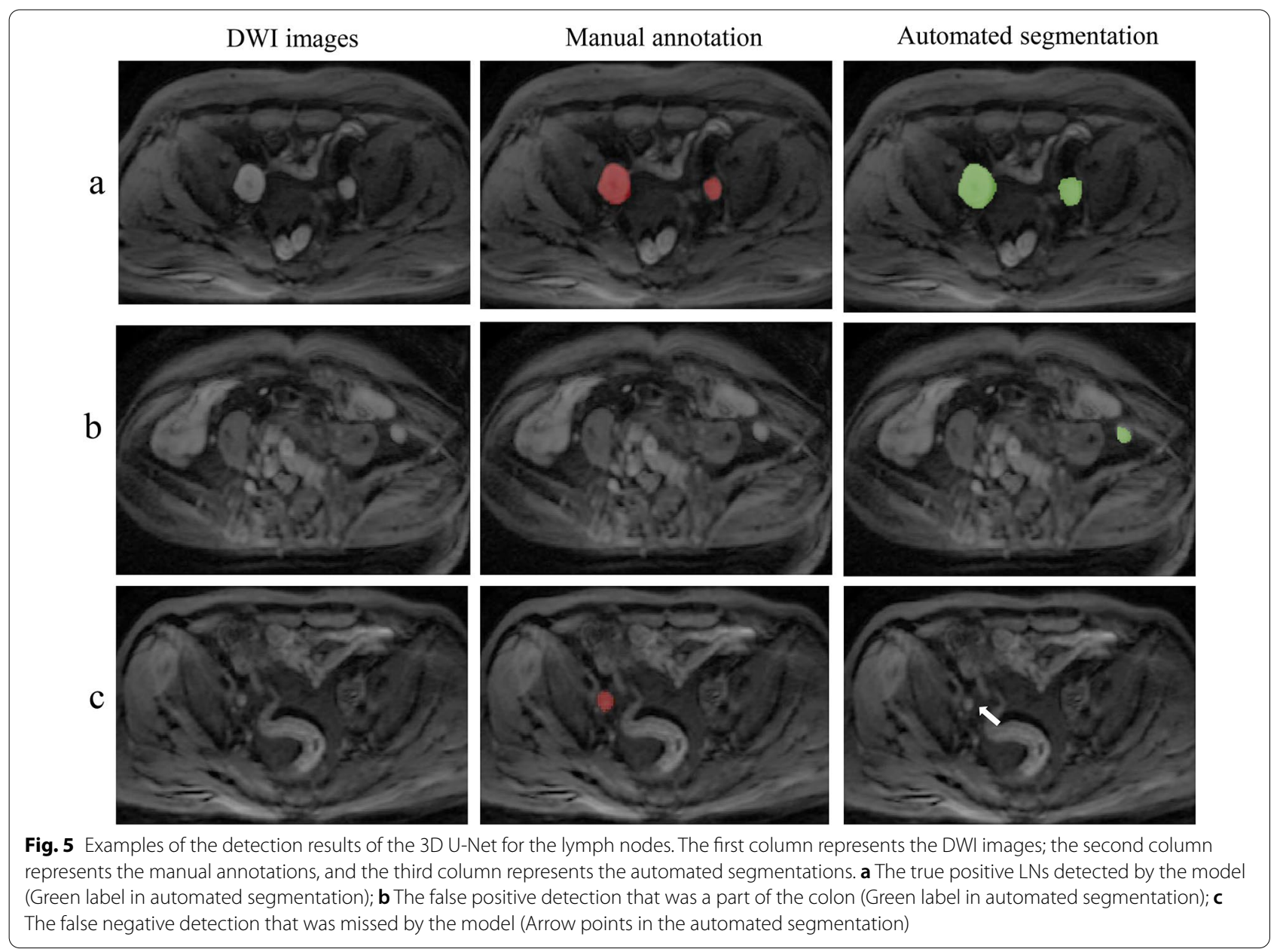

aorta where the anatomy of interest may be very small compared to the background consisting of connective tissue with a wide range of intensity grey values [26]. In this study, we used Dice as the loss function of the 3D U-Net algorithm and manually annotated all visible LNs in the algorithm development dataset, to obtain the specified voxel of the LNs as much as possible. Moreover, considering that LNs are nodular structures for which 3D information is helpful in distinguishing them from tubular structures, because both may show a blob-like structure in 2D images, the 3D U-Net model was selected for segmentation algorithm development [27]. To improve the efficiency and interoperator reliability of data annotation in this study, annotations performed by the two junior radiologists were corrected by an expert radiologist, and the two sets of annotations after modification achieved high Dice scores (Mask 3 vs. Mask 4: $0.88 \pm 0.06$ ) compared with those before correction (Mask 1 vs. Mask 2: $0.75 \pm 0.03)$. The results confirmed the reliability of manual annotation as ground truth for LN segmentation. It is fine to select either Mask 3 or Mask 4 as the ground truth. In this study, we choose Mask 4 as ground truth for the assessment of segmentation for which was performed later than Mask 3.

The algorithm in this study was trained for segmenting all visible LNs on DWI images, whether healthy or metastatic. Given that LNs with a short diameter of more than $0.8 \mathrm{~cm}$ were considered to be suspicious for metastasis and of more clinical significance, a cut-off threshold was set to filter out contiguous structures with a short diameter of less than $0.8 \mathrm{~cm}$. These selected LNs were used for the performance assessment of suspicious metastatic LNs. In clinical practice, radiologists usually measure and record the short diameter and volume of the largest LN instead of all metastatic LNs. Therefore, in this study, we also analysed the detection and segmentation performance of the model for the largest LNs. The N-staging was automatically generated based on the quantitative measurements (short diameter and volume) of the largest $\mathrm{LN}$ and was sent to the structured report of PCa.

Our results showed that the model achieved good segmentation accuracy of pelvic LNs with an average Dice 
score and VS of $0.76 \pm 0.15$ and $0.82 \pm 0.14$, respectively, in the testing set. Furthermore, the segmentation accuracy of suspicious LNs was significantly higher than that of all LNs (Dice score: 0.85 vs. 0.76, $P=0.009$; VS: 0.82 vs. $0.86, P=0.046$ ), which indicated that the segmentation model performed better with large LNs. The average short diameter and volume of the LNs were also measured to quantitively evaluate the segmentation performance. A short diameter is regarded as the reference value to determine the existence of suspicious metastatic LNs, and volume is of high significance for the evaluation of tumour load and response treatment. In our results, both the short diameter and volume of the automated segmentations showed a close correlation with manual annotations on all LNs, suspicious LNs and the largest LNs (all with $\mathrm{R}>0.80$ ).

To evaluate the segmentation performance of the model, except for the commonly used overlap-based metrics (Dice, TPR and PPV), the volume-based metric (VS), Spatial distance based metrics (HD, AVD, and MHD) were also defined to assess the segmentation accuracy of the 3D U-Net model. The Dice coefficient, which directly compares the overlap between automated segmentation and manual annotation, is the most commonly used metric for evaluating medical image segmentation [28]. The TPR measures the portion of positive voxels in the manual annotations that are also identified as positive by the automated segmentation, and the PPV indicates the proportion of positive voxels in the manual annotation to the positive voxels in the automated segmentation. VS is a measurement that indicates similarity and considers the volumes of manual segmentation and automated segmentation [21, 29]. With a high VS, the model might be an accurate and convenient tool to assess tumour burden in LNs. The HD and AVD are usually used for contour evaluation. Besides, the AVD is thought to be more stable and less sensitive to outliers than the HD. MHD is a metric for the evaluation of general shape and alignment, which takes into account the correlation of all points in the LNs[21].

The lesion detection approach is proposed based on the segmentation result obtained with the 3D U-Net model. Unlike the segmentation assessment, the detection assessment of LNs was focused on the suspicious LNs in the testing set and hold-out test dataset. This is because the detection of small LNs (short diameter $<0.8 \mathrm{~cm}$ ) is of little clinical significance. In the hold-out test dataset, in addition to the detection and segmentation assessment of 3D U-Net, we evaluated the clinical application value of the model in the evaluation of suspicious LNs by comparing the consistency of LN staging (N0 or N1) between different readers. Cohen's kappa coefficient between the model and expert radiologist was significantly higher than that between the resident and expert radiologist (0.922 vs. 0.844), which confirmed the feasibility of its clinical application and the possibility of becoming a promising tool for improving the diagnostic accuracy of LN staging for less experienced residents.

Hitesh et al. conducted similar research using U-Net for mediastinal and cervical LNs in CT images. Further, they achieved the differential diagnosis of malignant and benign mediastinal LNs with a fully convolutional network [30] and improved the performance using generative adversarial network and Inception network [31]. Their proposed FCN model has achieved an average sensitivity for the diagnosis of malignant LNs of $90.63 \%$ and was then increased to $94.95 \%$ by the GAN and Inception network. In our study, we used a fairly large dataset to train a 3D U-Net model and validated the model in a hold-out dataset, the model achieved a high recall (0.98) value in the detection of the suspicious LNs. Our results confirmed the feasibility of the 3D U-Net on automated detection and segmentation of pelvic LNs on DWI images. While some other deep learning approaches (e.g., ENet) $[13,32]$ may perform better than U-Net. In the future, we would compare the efficiency of these methods regarding the segmentation and detection of LNs.

Serval limitations need to be pointed out in this study. First, the current algorithm cannot provide the exact anatomical location of a particular LN, and a future $\mathrm{CNN}$ trained with multiclass annotations of different regional LNs (obturator nodes, external iliac nodes, internal iliac nodes, and common iliac nodes) may be helpful for anatomical location. Second, the 3D U-Net detects, segments and measures LNs larger than $0.8 \mathrm{~cm}$ in the short axis but does not render a diagnosis of metastatic LNs. Here, LNs suspicious of metastasis were diagnosed based on their short diameter, which is of reference value to some extent but cannot represent true metastatic LNs. More LN information from PET/ CT, MR lymphography or pelvic LN dissection may be necessary for reliable metastatic LN diagnosis. Third, we confined our proof-of-concept study to the pelvic area, but it should be extended to the whole body in the future. A further step towards a faster and comprehensive automated analysis that provides a global tumour burden in PCa patients is the detection and quantification of PCa lesions and skeletal metastases. In addition, although the high Dice score between Mask 3 and Mask 4 indicates that either Mask3 or Mask 4 is acceptable to be the ground truth for the assessment of segmentation, a STAPLE tool may be more convincing to establish the simultaneous ground truth by combining the different segmentations from the clinical experts in a consolidated reference [33]. In the future, we will consider applying the STAPLE tool for ground truth estimation 
on other segmentation tasks. Last, more data inclusion is necessary for construction of a more robust segmentation model, and multicentre data should be collected to further consolidate the generalization ability of our model. Only patients with PCa were included here, which potentially limits the transferability of our $\mathrm{CNN}$ to a broad range of bone metastases of other primary tumors (rectal cancer, bladder cancer, etc.). The application of this method for other types of LNs is needed in the scope of future work.

In conclusion, this study confirmed the feasibility of the 3D U-Net CNN for automated detection and segmentation of LNs on pelvic DWI images. This may present a promising step towards a clinically helpful deep learning-based tool that can provide a comprehensive and objective assessment of tumour burden in $\mathrm{PCa}$ patients.

\begin{abstract}
Abbreviations
AUC: Area under the curve; CNN: Convolutional neural network; DWl: Diffusion-weighted imaging; FP: False positive; FN: False negative; LNs: Lymph nodes; mpMRI: Multiparametric MRI; PCa: Prostate cancer; PLND: Pelvic lymph node dissection; PPV: Positive predictive value; TN: True negative; TPR: True positive rate; TP: True positive; VS: Volumetric similarity.
\end{abstract}

\section{Acknowledgements}

Not applicable.

\begin{abstract}
Authors' contributions
All authors contributed to the study conception and design. Material preparation, data collection and analysis were performed by $\mathrm{XL}, \mathrm{ZS}, \mathrm{CH}$ and $\mathrm{YC}$. XL and ZS performed manual annotation under the supervision of XW. JH, XW and XZ performed data interpretation and statistical analysis. The first draft of the manuscript was written by $X \mathrm{~L}$ and all authors commented on previous versions of the manuscript. All authors read and approved the final manuscript.
\end{abstract}

\section{Funding}

Capital's Funds for Health Improvement and Research (2020-2-40710).

\section{Availability of data and materials}

The datasets used and/or analyzed during the current study are available from the corresponding author on reasonable request.

\section{Code availability}

The code used for the development the algorithm are available from the corresponding author on reasonable request.

\section{Declarations}

\section{Ethics approval and consent to participate}

This study was performed in accordance with the principles of the Declaration of Helsinki and was approved by the Committee for Medical Ethics, Peking University First Hospital (2021-060). Written informed consent was obtained from all patients.

\section{Consent for publication}

Not applicable.

\section{Competing interests}

Jiahao Huang and Xiangpeng Wang are from a medical technical corporation provided technical support for model development. The authors declare that they have no competing interests.

\section{Author details}

${ }^{1}$ Department of Radiology, Peking University First Hospital, No.8 Xishiku Street, Xicheng District, Beijing 100034, China. ${ }^{2}$ Beijing Smart Tree Medical Technology Co. Ltd., No.24, Huangsi Street, Xicheng District, Beijing 100011, China.

Received: 5 October 2021 Accepted: 8 November 2021

Published online: 13 November 2021

\section{References}

1. von Bodman C, Godoy G, Chade DC, Cronin A, Tafe L, Fine SW, Laudone V, Scardino PT, Eastham JA. Predicting biochemical recurrence-free survival for patients with positive pelvic lymph nodes at radical prostatectomy. J Urol. 2010;184(1):143-8.

2. Kim YJ, Song C, Eom KY, Kim IA, Kim JS. Lymph node ratio determines the benefit of adjuvant radiotherapy in pathologically 3 or less lymph node-positive prostate cancer after radical prostatectomy: a population-based analysis with propensity-score matching. Oncotarget. 2017:8(66):110625-34.

3. Mottet N, Bellmunt J, Bolla M, Briers E, Cumberbatch MG, De Santis M, Fossati N, Gross T, Henry AM, Joniau S, et al. EAU-ESTRO-SIOG Guidelines on Prostate Cancer. Part 1: screening, diagnosis, and local treatment with curative intent. Eur Urol. 2017;71(4):618-29.

4. Gakis G, Boorjian SA, Briganti A, Joniau S, Karazanashvili G, Karnes RJ, Mattei A, Shariat SF, Stenzl A, Wirth M, et al. The role of radical prostatectomy and lymph node dissection in lymph node-positive prostate cancer: a systematic review of the literature. Eur Urol. 2014;66(2):191-9.

5. Hou Y, Bao ML, Wu CJ, Zhang J, Zhang YD, Shi HB. A machine learningassisted decision-support model to better identify patients with prostate cancer requiring an extended pelvic lymph node dissection. BJU Int. 2019;124(6):972-83.

6. Yakar D, Debats OA, Bomers JG, Schouten MG, Vos PC, van Lin E, Fütterer $\mathrm{J}$, Barentsz JO. Predictive value of MRI in the localization, staging, volume estimation, assessment of aggressiveness, and quidance of radiotherapy and biopsies in prostate cancer. J Magn Reson Imaging. 2012;35(1):20-31.

7. Perez-Lopez R, Tunariu N, Padhani AR, Oyen WJG, Fanti S, Vargas HA, Omlin A, Morris MJ, de Bono J, Koh DM. Imaging diagnosis and follow-up of advanced prostate cancer: clinical perspectives and state of the art. Radiology. 2019;292(2):273-86.

8. Hövels AM, Heesakkers RA, Adang EM, Jager GJ, Strum S, Hoogeveen YL, Severens JL, Barentsz JO. The diagnostic accuracy of CT and MRI in the staging of pelvic lymph nodes in patients with prostate cancer: a metaanalysis. Clin Radiol. 2008;63(4):387-95.

9. Woo S, Suh CH, Kim SY, Cho JY, Kim SH. The diagnostic performance of mri for detection of lymph node metastasis in bladder and prostate cancer: an updated systematic review and diagnostic meta-analysis. AJR Am J Roentgenol. 2018;210(3):W95-w109.

10. Sankineni S, Brown AM, Fascelli M, Law YM, Pinto PA, Choyke PL, Turkbey B. Lymph node staging in prostate cancer. Curr Urol Rep. 2015;16(5):30.

11. Litjens G, Kooi T, Bejnordi BE, Setio AAA, Ciompi F, Ghafoorian M, van der Laak J, van Ginneken B, Sánchez CI. A survey on deep learning in medical image analysis. Med Image Anal. 2017:42:60-88.

12. Chan HP, Samala RK, Hadjiiski LM, Zhou C. Deep learning in medical image analysis. Adv Exp Med Biol. 2020;1213:3-21.

13. Cuocolo R, Comelli A, Stefano A, Benfante V, Dahiya N, Stanzione A, Castaldo A, De Lucia DR, Yezzi A, Imbriaco M. Deep learning whole-gland and zonal prostate segmentation on a public MRI dataset. J Magn Reson Imaging 2021.

14. Comelli A, Coronnello C, Dahiya N, Benfante V, Palmucci S, Basile A, Vancheri C, Russo G, Yezzi A, Stefano A. Lung segmentation on highresolution computerized tomography images using deep learning: a preliminary step for radiomics studies. J Imaging 2020;6(11).

15. Roth HR, Lu L, Seff A, Cherry KM, Hoffman J, Wang S, Liu J, Turkbey E, Summers RM: A new 2.5D representation for lymph node detection using random sets of deep convolutional neural network observations. Medical image computing and computer-assisted intervention : MICCAI International Conference on Medical Image Computing and Computer-Assisted Intervention 2014, 17(Pt 1):520-527. 
16. Roth HR, Lu L, Liu J, Yao J, Seff A, Cherry K, Kim L, Summers RM. Improving computer-aided detection using convolutional neural networks and random view aggregation. IEEE Trans Med Imaging. 2016;35(5):1170-81.

17. Borrelli $P$, Larsson $M$, Ulén J, Enqvist $O$, Trägårdh $E$, Poulsen $M H$, Mortensen MA, Kjölhede H, Høilund-Carlsen PF, Edenbrandt L. Artificial intelligencebased detection of lymph node metastases by PET/CT predicts prostate cancer-specific survival. Clin Physiol Funct Imaging. 2021;41(1):62-7.

18. Zhao Y, Gafita A, Vollnberg B, Tetteh G, Haupt F, Afshar-Oromieh A, Menze B, Eiber M, Rominger A, Shi K. Deep neural network for automatic characterization of lesions on (68)Ga-PSMA-11 PET/CT. Eur J Nucl Med Mol Imaging. 2020;47(3):603-13.

19. Debats OA, Litjens GJ, Barentsz JO, Karssemeijer N, Huisman HJ. Automated 3-dimensional segmentation of pelvic lymph nodes in magnetic resonance images. Med Phys. 2011;38(11):6178-87.

20. Cicek O, Abdulkadir A, Lienkamp SS, Brox T, Ronneberger O: 3D U-Net: learning dense volumetric segmentation from sparse annotation. Medical Image Computing and Computer-Assisted Intervention - MICCAI 2016 19th International Conference Proceedings: LNCS 9901 2016:424-432.

21. Taha AA, Hanbury A. Metrics for evaluating 3D medical image segmentation: analysis, selection, and tool. BMC Med Imaging. 2015;15:29.

22. Ushinsky A, Bardis M, Glavis-Bloom J, Uchio E, Chantaduly C, Nguyentat M, Chow D, Chang PD, Houshyar R. A 3D-2D hybrid U-Net convolutional neural network approach to prostate organ segmentation of multiparametric MRI. AJR Am J Roentgenol. 2021;216(1):111-6.

23. Zhu Y, Wei R, Gao G, Ding L, Zhang X, Wang X, Zhang J. Fully automatic segmentation on prostate MR images based on cascaded fully convolution network. J Magn Reson Imaging. 2019;49(4):1149-56.

24. Chen Y, Xing L, Yu L, Bagshaw HP, Buyyounouski MK, Han B. Automatic intraprostatic lesion segmentation in multiparametric magnetic resonance images with proposed multiple branch UNet. Med Phys. 2020;47(12):6421-9.

25. De Fauw J, Ledsam JR, Romera-Paredes B, Nikolov S, Tomasev N, Blackwell S, Askham H, Glorot X, O'Donoghue B, Visentin D, et al. Clinically applicable deep learning for diagnosis and referral in retinal disease. Nat Med. 2018;24(9):1342-50.

26. Comelli A, Dahiya N, Stefano A, Benfante V, Gentile G, Agnese V, Raffa GM, Pilato M, Yezzi A, Petrucci G, et al. Deep learning approach for the segmentation of aneurysmal ascending aorta. Biomed Eng Lett. 2021;11(1):15-24.

27. Nemoto T, Futakami N, Yagi M, Kumabe A, Takeda A, Kunieda E, Shigematsu N. Efficacy evaluation of 2D, 3D U-Net semantic segmentation and atlas-based segmentation of normal lungs excluding the trachea and main bronchi. J Radiat Res. 2020;61(2):257-64.

28. Hwang D, Kim KY, Kang SK, Seo S, Paeng JC, Lee DS, Lee JS. Improving the accuracy of simultaneously reconstructed activity and attenuation maps using deep learning. J Nucl Med. 2018;59(10):1624-9.

29. Cárdenes R, de Luis-García R, Bach-Cuadra M. A multidimensional segmentation evaluation for medical image data. Comput Methods Programs Biomed. 2009;96(2):108-24.

30. Tekchandani $H$, Verma S, Londhe ND. Mediastinal lymph node malignancy detection in computed tomography images using fully convolutional network. Biocybern Biomed Eng. 2020;40(1):187-99.

31. Tekchandani H, Verma S, Londhe N. Performance improvement of mediastinal lymph node severity detection using GAN and Inception network. Comput Methods Programs Biomed. 2020;194:105478.

32. Stefano A, Comelli A. Customized efficient neural network for COVID-19 infected region identification in CT images. J Imaging. 2021;7(8):131.

33. Comelli A, Dahiya N, Stefano A, Vernuccio F, Portoghese M, Cutaia G, Bruno A, Salvaggio G, Yezzi A. Deep learning-based methods for prostate segmentation in magnetic resonance imaging. Appl Sci (Basel). 2021;11(2):782.

\section{Publisher's Note}

Springer Nature remains neutral with regard to jurisdictional claims in published maps and institutional affiliations.
Ready to submit your research? Choose BMC and benefit from:

- fast, convenient online submission

- thorough peer review by experienced researchers in your field

- rapid publication on acceptance

- support for research data, including large and complex data types

- gold Open Access which fosters wider collaboration and increased citations

- maximum visibility for your research: over $100 \mathrm{M}$ website views per year

At BMC, research is always in progress.

Learn more biomedcentral.com/submissions 